\section{¿DESCOLONIZAR INTERNAMENTE? RASTREANDO LOS PATRONES MILITARES Y COLONIZADORES EN ECUADOR A TRAVÉS DE LA GESTIÓN DE RIESGOS}

Johannes M. Waldmüller ${ }^{1^{*}}$ y María Susana Robledo ${ }^{2}$

\section{RESUMEN}

En Ecuador predomina un enfoque técnico-científico y militar en la gestión de riesgos, basado en la seguridad nacional y la respuesta inmediata al desastre. En base a dos casos históricos, la erupción del volcán Tungurahua (1999-2006) y del terremoto costeño (2016-2018), se debate la trayectoria colonial interna de la gestión de riesgos en Ecuador, subordinada a los intereses políticos y económicos de los gobiernos y élites de turno. Adicionalmente, se vislumbra la trayectoria que tomaron las instituciones, la producción de conocimiento "oficial" y el rol de las FF.AA. desde los años 90 con respecto a la gestión de riesgos. Si bien hubo ciertos cambios institucionales y otros avances, prevalece un sesgo en la atención de los riesgos y desastres que le ha restado a las poblaciones locales, indígenas campesinas y afroecuatorianos, la oportunidad de empoderarse en la gestión de su propio territorio y sus recursos económicos asignados, prologando formas del colonialismo interno, en clave étnica y de género. Se concluye que la reducción de la vulnerabilidad mediante un marco-legal adecuado, todavía ausente, implicaría que la población sea reconocida como un actor participante de educación, prevención y mitigación.

\section{PALABRAS CLAVES}

Ecuador, Gestión de riesgos, Descolonización, Tungurahua, Defensa Civil

\section{DECOLONIZE INTERNALLY? TRACKING THE MILITARY AND COLONIZING PATTERNS IN ECUADOR THROUGH RISK MANAGEMENT}

\begin{abstract}
A techno-scientific and military approach predominates risk management in Ecuador, focusing on national security and immediate response to disaster. Drawing from two historical cases, the eruption of the Tungurahua volcano (1999-2006) and the coastal earthquake (2016-2018), we discuss the internal colonial trajectory of risk management in Ecuador as being fundamentally subordinated to the political and economic interests of the governments and powerful. Additionally, we shed light on the trajectory taken by the concerned institutions, the production of "official" knowledge and the role of the Armed Forces. since the 90 s with regard to risk management. Although there have been certain institutional and policy advances, an important bias prevails in the attention to risks and disasters that continues to impact local populations, indigenous peasants and Afro-Ecuadorians, and their opportunity to empower themselves in the management of their own territory and their economic resources, equaling to prolonged forms of internal colonialism in ethnic and gendered dimensions. We conclude that the preventive reduction of vulnerability through an adequate legal framework, still absent today, would imply that vulnerable populations are recognized as relevant participating actors in education, prevention and mitigation.
\end{abstract}

\section{KEYWORDS}

Ecuador, Disaster Management, Decoloniality, Tungurahua, Civil Defense
1. Facultad de Ciencias Administrativas, Escuela Politécnica Nacional (EPN), Quito, Ecuador.

2. FLACSO, Quito, Ecuador.

*Autor de correspondencia: johannes.waldmuller@ graduateinstitute.ch

Identificador:

http://revistareder.com/ handle-0719-8477-2021-092

\section{RECIBIDO}

18 de febrero de 2021

\section{ACEPTADO}

5 de abril de 2021

\section{PUBLICADO}

1 de julio de 2021

\section{Formato cita}

Recomendada (APA): Waldmüller, J.M. \& Robledo, M.S. (2021). ¿Descolonizar internamente? Rastreando los patrones militares y colonizadores en Ecuador a través de la gestión de riesgos. Revista de Estudios Latinoamericanos sobre Reducción del Riesgo de Desastres REDER, 5(2), 3447. http://revistareder.com/ handle-0719-8477-2021-092

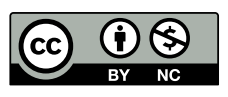

Todos los artículos publicados en REDER siguen una política de Acceso Abierto y se respaldan en una Licencia CreativeCommons Atribución-NoComercial 4.0 Internacional.

Revista de Estudios Latinoamericanos sobre Reducción del Riesgo de Desastres (REDER)

Diseño: Lupe Bezzina 


\section{INTRODUCCIÓN}

El presente artículo se inscribe en el amplio debate sobre la de(s)colonización del conocimiento y prácticas del desarrollo, discusiones que, actualmente, se encuentran más presentes en el norte global (por ej. Konyndyk \& Aly, 2021; Timmermann, 2020), en particular en el contexto de la actual pandemia vinculada al virus SARS-Covid-19. Sin embargo, en lo concreto, aquí se busca profundizar el debate desde una mirada interna e histórica del Sur con respecto a la gestión y respuesta a los eventos naturales (como antecedente importante para entender la pandemia actual). $Y$ en particular, en la región andina donde, si bien hubo algunos importantes avances en la gestión estatal en los últimos 15 a 20 años (Rebotier, 2016), se parte de la hipótesis de la prevalencia de un enfoque primordialmente técnico-científico relacionado al rápido "solucionismo" (Morozov, 2013), sumado a un sesgo modernista-colonial-urbano y de seguridad nacional (en términos militares) (Carrigan, 2015). Como se pretende demostrar mediante una reflexión con base en el material empírico acumulado desde hace varios años, en los países andinos tal enfoque tiende a relegar temas vitales como protocolos de prevención y de respuesta inmediata en situación de catástrofe, a expertos tecnocráticos y militares cuyas planificaciones no se acoplan con las demandas socioeconómicas de la población, entre ellas, igualdad social y transformación sostenible, etc.

Esta situación se refleja en la casi nula existencia de programas o currículos de estudios académicos en gestión de desastres en el área de las ciencias sociales (con las notables, aunque parciales, excepciones del CIGIDEN en Chile y FLACSO Costa Rica), es decir, programas basados en categorías sociales claves como cultura, historia, poder, clase social, etnia y género, como elementos transversales al análisis de la vulnerabilidad, la resiliencia y la exposición al riesgo, sumándose a la tercera ola poscolonial de discusión sobre comunidad y vulnerabilidad en un sentido estructural y reproductivo (Atallah, 2016; Atallah et al., 2019; Fernández et al., 2020).

Asimismo, se evidencia una importante falta de recursos, humanos y financieros, destinados a investigaciones regionales y globales, ante la realidad urgente representada en las nuevas amenazas antropogénicas, entre ellas, el cambio climático, la migración y la expansión del modelo rentista-extractivo en nuestra región, todas directamente vinculadas con la necesidad de nuevos modelos de desarrollo pos-neoliberal (Büscher et al., 2021).

En este contexto, resulta vital plantearse en clave histórica, cómo los procesos frecuentemente instrumentalizados de respuestas frente a desastres en nuestra región, han contribuido a profundizar formas de dominio técnico-científico, modernista-colonial y biopolítico (Marchezini, 2015) sobre las poblaciones y los territorios. Resulta importante rastrear y vislumbrar cómo este control contribuye a re-organizar o direccionar, bajo la mirada de élites dominantes, la terratenencia, el acceso a los recursos, o determinadas decisiones políticas y económicas según la mirada útil de aquellos expertos y perspectivas técnicas afines al poder de turno. Por tal fin, se hace necesario recurrir a teorías decoloniales (entre otros, Escobar, 2002; Moraña et al., 2008; Walsh, 2009) que permitan desarrollar perspectivas críticas sobre sus fundamentos.

En base a dos casos empíricos del Ecuador, analizados en detalle en varias publicaciones anteriores (Waldmueller, Nogales y Cobey, 2019; Waldmüller, Nogales, Cobey y Urigüen, 2018), así como una revisión de la literatura pertinente y de la cobertura mediática histórica, además de algunas entrevistas con informantes claves, se debate aquí la trayectoria internamente colonial de la gestión de riesgos en Ecuador que, al largo de los años, y corroborando nuestra hipótesis de partida, quedó subyugada a los intereses políticos y económicos de los gobiernos de turno. Ambos casos presentados son fruto de trabajo cualitativo de campo, realizado entre los años 2017 y 2020 , con más de 150 entrevistas en total, incluyendo observaciones, grupos focales y documentación visual, realizados con damnificados, políticos, expertos locales y representantes de las agencias multilaterales, algunos de ellos entrevistados en repetidas ocasiones.

El primer caso es la prolongada erupción del volcán Tungurahua (1999-2006), uno de los más activos en nuestro planeta, donde se presentan datos primarios novedosos con respecto a las drásticas medidas tomadas en relación a la población local, como la evacuación total y forzada de una de las ciudades turísticas más importantes del país. El segundo caso no presenta nuevos datos, sino una reflexión posterior sobre la mala gestión de las consecuencias del terremoto costeño del 2016, y que actualmente constituye uno de los episodios más corruptos en la historia del Ecuador (Ardaya Salina, 2019). 
Desde siempre, tanto el colonialismo histórico, como la condición sociocultural de la colonialidad poscolonial (Castro-Gómez, 2005; Mignolo, 2011; Escobar, 2002), han tenido dos caras: una hacia el exterior, en torno a los grandes poderes y sus esferas de influencia, y otra hacia el interior (Amin, 1974; Fanon, 1966, 1967; Lebret, 1967) que en el contexto desigual global, se conoce bajo la figura de "colonialismo interno" (González Casanova, 1965), expresado en aristas económicas, políticas sociales y culturales locales; y siempre bajo la esfera de acción del Estadonación y del capitalismo.

Ecuador, al ser un país expuesto a "fenómenos geológicos e hidrometeorológicos que se presentan con singular frecuencia y violencia (...) fenómenos naturales potencialmente destructivos, combinados con las acentuadas características de vulnerabilidad global" (Aguilera y Toulkeridis, 2005, p. 5) requiere de un sistema de gestión de riesgos que pueda dar respuestas inmediatas a la población en caso de eventuales sucesos naturales, así como políticas y prácticas eficaces de prevención y monitoreo constante.

No obstante, y al igual que en otros países de la región, hasta hace relativamente poco la gestión de riesgos por parte del Estado ha sido organizada en el marco de la Defensa Civil, bajo la lógica, formación y logística militar (Yépez et al., 2020), la cual incluyó una definición reducida de las amenazas y la seguridad (Andrade-Vásquez, 2021). Por ello, la gestión de riesgos se ha convertido en un tema complejo, atravesado por una serie de principios esencialmente jerárquicos, tecnológicos y científicos, principalmente enfocados en la respuesta técnica y logística inmediata al desastre, que deja de lado el incentivo de la cultura de la prevención y la acción comunitaria

Desde una perspectiva decolonial, y abordando la historia reciente de la gestión de riesgos en Ecuador, se entiende que, ante una amenaza concreta, se desencadenan diferentes agencialidades que implican la naturalización de jerarquías territoriales y sociales, ajenas a la identidad cultural de las poblaciones de las regiones afectadas. En este contexto, una perspectiva decolonial -en constante construcción- constituye "una propuesta epistémica, teórica y metodológica 'latinoamericana' para comprender las relaciones de poder/dominio en el espacio-tiempo, así como para la superación de la matriz histórica-colonial de poder y la liberación de los sujetos sub-alternos de esa matriz" (Vargas, 2009, p. 46). La perspectiva decolonial no se inscribe en el marco de las grandes teorías modernistas, como son el marxismo o el liberalismo, sino en oposición a tales "metarelatos" (Lyotard, 1979). Destaca la siempre presente estructura colonial implícita u oculta, presente en todo proceso de modernización, la cual no hace referencia al episodio histórico del colonialismo, sino a la condición de la colonialidad en términos de jerarquías en las formas de conocimiento, las distintas experiencias identitarias (etnia, religión, fenotipos) y la perspectiva de género, con la prevalencia de una matriz colonial de poder (Walsh, 2004).

Ahora bien, con respecto al "colonialismo interno", no se busca profundizar únicamente en las relaciones globales y geopolíticas de poder que sujetan a los países en vía de desarrollo, sino entender la reproducción de tales formas de dominación al interior de aquellos países y de comunidades locales, donde frecuentemente, como en el caso de nuestra región, se jerarquizan y perciben conocimientos occidentales "científicos" como superiores a los conocimientos "ancestrales" indígenas, o la masculinidad blanca superior a la feminidad afrodescendiente, y por supuesto, donde ciertos sectores y clases sociales se suman "estructuralmente por encima a través de la explotación de otros grupos culturalmente heterogéneos y diferentes" (González Casanova, 1965, p. 33)

En relación a ello, se presenta, primeramente, un breve resumen de la gestión de riesgos y su trayectoria cambiante en el país. El mismo genera varias preguntas respecto al presente y futuro de la gestión y generación de conocimientos técnico-científicos "validos" que se retomará en el apartado final nuevamente. En segundo lugar, se describen los acontecimientos económicos y políticos más destacados de los años 90 y 2000. A la luz de aquellos, se analiza, en tercer lugar, el tratamiento de los riesgos asociados al estallido del volcán Tungurahua, ubicado en la provincia ecuatoriana del mismo nombre, que entre el 1999 y 2006 se encontró en un proceso eruptivo. Durante este período se sucedieron acontecimientos que representan la capacidad de gestionar un escenario catastrófico e inesperado por parte del Estado ecuatoriano, mediante el despliegue del ejército y el desplazamiento forzado de la población ubicada al pie del volcán, tanto de la ciudad de Baños de Agua Santa, como de comunidades rurales cercanas, acciones que pueden considerarse un prototipo de las lógicas coloniales internas en la gestión de riesgos. 
Adicionalmente, tales hechos permitieron encubrir otras acciones significativas del Estado en aquella época, algunas con repercusiones hasta el día de hoy, como la crisis política de entonces, plasmada en la sucesión de gobiernos en poco tiempo, la dolarización del año 2000, y el empobrecimiento acelerado de la sociedad ecuatoriana debido a ajustes estructurales bajo el "consenso de Washington", la liberalización del sector financiero bajo el expresidente Durán-Ballén y el congelamiento de las cuentas bancarias (a inicios de 1999) en el contexto de la conversión forzada del Sucre al dólar estadounidense como moneda nacional. En el contexto global se sucedieron acontecimientos que tuvieron una gran influencia en América Latina, entre ellos se destacan la caída del precio del petróleo (1998-1999), la crisis económica internacional iniciada en Asia en 1997, y de forma particular, el fenómeno de El Niño en 1998, que causó tremendos daños en la economía costeña ecuatoriana. Asimismo, es de destacar que, en 1997, el movimiento indígena nacional fue un importante protagonista en el derrocamiento del gobierno de Abdalá Bucaram, percibido como altamente corrupto. Más tarde, en el año 2000, nuevamente la Confederación de Nacionalidades Indígenas de Ecuador (CONAIE) lideró la destitución del gobierno de Jamil Mahuad, presidente en funciones al momento de iniciarse la erupción del Tungurahua.

Planteamos, como cuarto punto, que el tratamiento de los afectados con la erupción del volcán, en la Sierra, presenta un antecedente en el enfoque público y militar desplegado también ante el terremoto de abril de 2016, que devastó las provincias costeñas de Manabí, Esmeraldas y Santa Elena. En consecuencia, la respuesta fue, nuevamente, el masivo y forzado desplazamiento de miles de personas, primero a albergues bajo control militar, y luego de un año o más, hacia la re-ubicación de los damnificados en viviendas públicas de mínima extensión y deplorable calidad (Waldmüller et al. 2018; Waldmueller, et. al. 2019).

Comprendiendo que la perspectiva decolonial busca revalorizar las experiencias locales sobre el territorio y sus prácticas cotidianas, se propone la reflexión sobre las lógicas del accionar institucional en relación con la tendencia a la creciente militarización de la región y el inmenso incremento del gasto militar a partir del 9/11, lo cual implica una reorientación de las amenazas y riesgos hacia la misma sociedad civil interna, ahora vista como potenciales enemigos destabilizantes (Müller, 2020). Se concluye arguyendo que la gestión de riesgos en Ecuador, de forma similar a otros países de la región y en total discrepancia con las declaraciones gubernamentales e internacionales, profundizó las lógicas del colonialismo interno durante los últimos 20 años. Si bien el género en sí representa un marcador social y perspectiva epistémica muy relevante para el análisis de tal profundización, en este artículo se aborda de forma interseccional (Collins \& Bilge, 2016; Crenshaw, 1989). Es decir, respecto al impacto de la gestión estatal de riesgo, se observa el cruce de varios marcadores sociales para demostrar como las políticas públicas aplicadas afectan a las poblaciones más vulnerables, o internamente colonizadas, incluyendo con más frecuencia a las mujeres. En la siguiente sección se abordarán orientaciones esenciales que inciden en la gestión de riesgos en el país, seguidas de una contextualización económica y política de la época relevante para el argumento principal; finalmente discutimos los casos de estudio.

\section{POLÍTICA DE GESTIÓN DE RIESGOS (GDR) EN ECUADOR}

Siendo relevante para la discusión en adelante, esta sección rastrea los lineamientos básicos y cambiantes de la GdR en Ecuador, que moldean su institucionalidad siempre transitoria, la cual responde a los contextos dinámicos internacionales y regionales de la $\mathrm{GdR}$ en las últimas décadas (Hannigan, 2012; Watson, 2019) .

Para empezar, y de acuerdo con Rodriguez y Toulkeridis (2019), la actividad sísmica en el Ecuador se debe "a los movimientos bruscos que se dan encima o cerca de fallamiento de rocas 0 depósitos de rocas, generados por la deriva de las grandes placas existentes alrededor del país" (p. 45), lo cual se refleja en los acontecimientos recurrentes de movimientos y temblores. Por su parte, D'Ercole y Trujillo (2003) entienden que, dada la ubicación del Ecuador este tipo de fenómenos han provocado un gran desequilibrio socioeconómico y ambiental, registrándose más de 15.000 personas fallecidas y 4 millones de afectados durante los eventos catastróficos que se sucedieron solo en el siglo XX, entre estos se pueden contar terremotos, erupciones, tsunamis, inundaciones y deslizamientos.

En respuesta a ello, en 1973 comenzó a operar plenamente la Defensa Civil, cuyo objetivo fue la prevención de desastres, por lo cual iniciaron una serie de tareas en diferentes comunidades consideradas vulnerables, si bien tuvieron por objetivo la prevención, su acción más importante 
se registra en relación a las políticas públicas implementadas luego de sucedidos eventos extraordinarios, volviéndose más operativa (Villamarin, Grunauer y Mosquera, 2017). Toulkeridis, Bernabé-Poveda y Baile, et al. (2015) describen este momento como de gran autonomía para la fuerza militar, dado que encabezaban el Gobierno y se encontraban a cargo de la seguridad nacional.

Por otra parte, diversos estudios coinciden en la relación directa entre vulnerabilidad, riesgos y pobreza (Trelles y Donoso, 2018; D'Ercole y Trujillo, 2003; Corominas y Martí, 2015), relacionados a factores claves, como la exposición a los mismos, dado que la capacidad de anticipación, resistencia y recuperación de la población ecuatoriana debe analizarse en vista de componentes como acceso al agua potable y saneamiento, nivel de salud y educación, teniendo en cuenta marcadores sociales como la edad, etnia y clases sociales, además de una perspectiva de género, así como de seguridad alimentaria, ello en relación con niveles de pobreza y desarrollo (Cardona, 2008).

El análisis de la ubicación de la población, donde la incidencia de estas variables son más altas, coincide frecuentemente con las zonas más propensas a acontecimientos catastróficos, es decir, que la seguridad en relación con el acceso al agua y alimento se ve disminuida entre las comunidades más vulnerables, donde las mujeres se ven incluso más expuestas, tratándose de un sector históricamente relegado de las políticas de acceso a la educación y, tradicionalmente, responsables de la seguridad alimentaria de las familias, así como del cuidado familiar y comunitario posdesastre (Fernández et al., 2020; Vega et al., 2019). Por ejemplo, las mujeres son quienes que, en gran medida y a pesar de la falta de visibilizar el trabajo doméstico y comunitario, se encargan del cuidado de plantaciones, ancianos, animales y de la alimentación, pero también de la socialización de niños, niñas, como fundamento constituyente de la reproducción laboral en el sistema económico capitalista (Cielo \& Vega, 2015; Federici, 2014; Kofman, 2016).

De acuerdo a las lógicas internacionales y regionales de la $\mathrm{GdR}$, la política de gestión de riesgos en Ecuador se fue modificando también. Una fuerte cambio normativo puede identificarse desde 1960, con la introducción de las primeras normas legales correspondientes a la Dirección Nacional de Defensa Civil que dependía del Consejo de Seguridad Nacional (COSENA) y del Presidente, y que tuvo fuertes problemas de presupuesto. En 1997, pasada la dictadura militar, se creó la Unidad Coordinadora de Emergencia, y en 1998 la Corporación Ejecutiva para la Reconstrucción de las Zonas afectadas por el Fenómeno de El Niño (CORPOECUADOR) (Plan Operativo General, POG, 2005). En mayo de 1998 se reforma la antigua Dirección Nacional de Defensa Civil en una Secretaría Técnica de Gestión de Riesgos suscrita al Ministerio Coordinador de Seguridad Interna y Externa. En 1999 se inaugura la Oficina Nacional de Planificación (ODEPLAN), luego conocida como Secretaría Nacional de Planificación (SENPLADES), cuyo objetivo inicial fue diseñar planes de desarrollo que consideraran el riesgo ante eventos naturales.

En grandes líneas, y correspondiendo las lógicas internacionales que favorecieron las respuestas humanitarias y modernistas en lugar de la mitigación y prevención (Watson, 2019), estos organismos pertenecieron al ámbito de la Seguridad Nacional, y tuvieron por objetivo generar acciones de prevención y modernizar un sistema nacional de alerta temprana a la amenaza (POG, 2005), siempre en el marco de la seguridad entendida como orden y estabilidad. A pesar de ello, las transformaciones institucionales no lograron anticipar ni amortiguar el impacto del contexto económico y político que provocó, a finales de la década del 90 , una profunda crisis en el país. Con el objetivo de arribar a una mayor comprensión de lo sucedido, tales acontecimientos se presentan en la siguiente sección.

\section{CONTEXTO POLÍTICO Y CRISIS ECONÓMICA EN LA DÉCADA DE 1990}

En búsqueda de una estabilidad macroeconómica, y pasado el auge petrolero, Ecuador impulsó desde la década de los 80 una serie de políticas de ajuste estructural, siguiendo las recomendaciones del provenientes del Consenso de Washington (Williamson, 1993), las cuales estaban orientadas a reducir el gasto público social e incentivar la privatización de empresas públicas (Báez Flores, 2015). Cuatro gobiernos (1984 a 1996) siguieron las indicaciones de entidades internacionales a pie ${ }^{1}$, en particular del Fondo Monetario Internacional (FMI). Entre las diversas decisiones de política económica se liberaron los tipos de cambio y de interés, los controles y monitoreo en el sector financiero (García Osorio, 2013), se desarmó la protección arancelaria y laboral, se abrieron los mercados, se eliminaron subsidios y se implementaron medidas de desregularización del sistema financiero (Larrea, 2004). 
El aumento de la pobreza en la región fue estrepitoso, razón por la cual se implementaron diversos programas de ayuda social denominados "Fondos de Desarrollo Social". En 1990, en Ecuador se crea el "Fondo de Inversión Social para Emergencias" (FISE) y posteriormente en 1993 se aprobó la Ley de Modernización del Estado, Privatizaciones y Prestación de Servicios Públicos por parte de la Iniciativa Privada, la cual, a través de la Ley de Creación del Fondo de Solidaridad (FS) tuvo la intención de utilizar recursos provenientes de la privatización de empresas públicas en la implementación de programas de educación, salud, desarrollo humano, preservación del medio ambiente, construcción de viviendas sociales, etc., planificación orientada hacia los sectores más vulnerables del Ecuador (Báez Flores, 2015). Para Bustos (1999) los Planes de Desarrollo Social fueron clientelistas y corporativistas, ejecutados por el Estado de forma incorrecta, dado que el Plan FISE nunca tuvo incidencia real en la gestión de los territorios, ni en los problemas concretos de las poblaciones vulnerables. Si bien no fue un plan pensado en la gestión de riesgos, si tuvo por objetivo trabajar en reducir las variables de vulnerabilidad de las poblaciones más empobrecidas del Ecuador.

Sin embargo, los problemas estructurales de la economía primaria ecuatoriana, tales como una baja diversificación de exportaciones, una clase dominante rentista y deficiencias en la institucionalidad, sumados a cuestiones históricas como inequidad social y la exclusión, propiciaron que, en 1995 el índice de pobreza alcanzara un $56 \%$ de la población, del cual un $76 \%$ correspondió al área rural, inevitablemente, a finales de la década sobrevino una profunda crisis económica, social y política. A la crisis interna, se sumó un contexto socioeconómicamente difícil, marcado por el fenómeno de El Niño en 1997/1998, la caída de los precios del petróleo y la crisis financiera internacional (Larrea, 2004).

La poca incidencia del plan FISE en la gestión territorial pudo deberse a la crisis económica y política general que atravesó al Estado y que abrió al país y la sociedad a diversas formas de cooperación al desarrollo e influencia a través de ONGs internacionales (Andolina et al., 2009). Estas transformaciones se plasmaron luego de la elección como presidente, en 1998, de Jorge Jamil Mahuad Witt, quien heredó una aguda crisis financiera que sucesivamente empeoró. Otro elemento importante es el fenómeno de El Niño, ya mencionado anteriormente, puesto que afectó a los productores agroexportadores de la Costa, que no pudieron cubrir sus créditos con los bancos. En 1998 cae el precio internacional del petróleo, lo que profundizó los problemas fiscales y la balanza comercial. En resumen, la crisis bancaria tiene su origen en estos cambios macroeconómicos, en cuya base se encuentra la falta de control local sobre el sistema financiero, la incertidumbre política y la ausencia de una política monetaria fuerte que pudiera resistir frente a los sucesos internacionales.

Al proceso de devaluación constante se sumó a la inestabilidad política, lo cual generó el retiro de fondos de los ahorristas. Los intentos de estabilizar la economía no fueron efectivos y el precio del dólar no paró de aumentar, mientras el aparato productivo se encontraba, en parte, paralizado. Todos estos elementos convergen y se calcula que la inflación pasó de $43,3 \%$ en 1998, a 90\% en el primer año de dolarización, en el 2000. Como indicadores de la contracción de la economía se tiene el aumento del desempleo (más de 15\% en 1999), reducción de la inversión extranjera, devaluación de la moneda nacional, y la relación entre deuda externa y PIB $(100 \%$ en 1999).

El sistema bancario registró un aumento de cartera vencida, en un contexto de fuerte inflación y caída del consumo. En un intento de frenar la recesión, el 9 de enero del 2000 se dolariza oficialmente la economía, esto benefició al sistema financiero, al sector de la construcción, a la recaudación del gobierno y a los importadores, y tuvo consecuencias negativas para productores y exportadores, pequeña y mediana empresa, y la población económicamente activa.

La fecha es relevante, ya que pocos días antes los habitantes de Baños de Santa Agua y sus recintos rurales se tomaron su ciudad por la fuerza, en conflicto abierto con los militares. Ubicándose en la única ruta relativamente rápida que en ese entonces conectaba con la región sureña de la Amazonía ecuatoriana, en particular las ciudades de Puyo y Macas con la Sierra y vía a Quito, tanto el cierre de la ruta por los militares y el volcán, como la toma y apertura de la misma fueron, como argumentamos en adelante, acciones estratégicas. Algunos de los motivos se encuentran en los antecedentes históricos de la CONAIE, entre ellos las marchas hacia Quito y la derrota del anterior presidente, con el agregado de la participación activa de nacionalidades 
indígenas amazónicas, conocidas por su valentía y experiencia en tácticas de guerrilla como resultado de su participación en la guerra del Cenepa contra el Perú en 1995.

A continuación, se presentan los casos de estudio y el hilo que los conecta en términos de secuencia histórica y constitución de antecedentes para la GdR.

\section{PATRONES DE COLONIZACIÓN INTERNA: ERUPCIÓN DEL VOLCÁN TUNGURAHUA (1999- 2006) Y TERREMOTO DEL LITORAL (2016)}

El volcán Tungurahua se encuentra en la Cordillera Real de Ecuador, sobre el límite de las provincias de Chimborazo y Tungurahua, tiene una altura de 5020 metros y se ubica a $120 \mathrm{~km}$ al sur de Quito, $33 \mathrm{~km}$ de Ambato y a $8 \mathrm{~km}$ al Norte de la ciudad de Baños de Agua Santa, uno de los destinos turísticos más importantes del país. En el año 1999, el Tungurahua entró en erupción, ante ello, las autoridades declararon "alerta naranja", provocando una evacuación forzada de la población - aproximadamente 12.000 (GAD Baños de Agua Santa, 2014) - el 16 de octubre de 1999. Si bien en la ciudad nunca cayeron cenizas, quedó cuatro meses vacía, saqueada por militares insuficientemente aprovisionados, y generando un enorme impacto social y económico (Lane, Tobin y Whiterford, 2003; Tobin y Whiterford, 2002).

Para la ciudad de Baños de Agua Santa, la alerta naranja declarada por el gobierno representó la llegada de militares con la orden de evacuar el pueblo, si bien con resistencias, los pobladores abandonaron sus casas y su ganado hasta el momento en que regresaron (05 de enero de 2000) expulsando a los mismos militares en un violento enfrentamiento?

A nivel gubernamental, se implementaron planes de reubicación de la población campesina, realizados de forma abrupta y sin una planificación adecuada, así como la asignación de recursos económicos que no se han visto plasmados en ayuda concreta o planes de prevención. Según el exalcalde J.L. Freire, (comunicación personal, 6 de julio 2020, en Baños):

"Más o menos para septiembre llega defensa civil que llegó a ser un ente odiado acá, y empiezan a capacitar dicen que el volcán se puede activar, pero nunca dicen que ya estaba en actividad, y empiezan a capacitar con la película 'Volcano', y reunían a la gente en las casas comunales, en las escuelas, en las iglesias y decían "esto es lo que puede pasar" si a eso le sumas que toda la vida nos dijeron que cuando esto pase nos vamos a morir, la gente entra en un estado de pánico y qué hacemos, y el alcalde asustado, el geo físico asustado, los niños asustados, el presidente Mahuad, que estaba en una crisis económica terrible[...]"

Igualmente, para el campo científico implicó ciertas luchas epistémicas con base en el conocimiento científico-técnico y la apropiación del discurso del riesgo, entre quienes afirman que la evacuación de Baños de Agua Santa no fue necesaria (incluyendo la iglesia católica local), y quienes consideran que si fue preciso movilizar a todo un pueblo.

Se impuso la visión del Instituto Geofísico (IG), de la Escuela Politécnica Nacional (EPN), que hasta el día de hoy suministra, de manera privilegiada y sujeto a costos, a las instituciones estatales los datos y análisis, desde las ciencias técnicas, en materia de riesgos. Si bien el IG desplazó de alguna manera a la Universidad de las Fuerzas Armadas (ESPE) en la generación de datos oficiales, este proceso no ha sido sin roces con efectos potencialmente muy dramáticos para la población general. Por ejemplo, en los casos de las erupciones de los volcanes Cotopaxi (2015) y Pichincha (1999), en las cercanías de Quito, que cuenta con aproximadamente dos millones de habitantes, los análisis sobre el alcance de la amenaza, exposición y medidas a tomar por parte de la política variaron considerablemente.

De igual manera, la población local de Baños no consideró necesario evacuar su ciudad, tanto por la falta de antecedentes históricos de gravedad, como por sus conocimientos ancestrales sobre los flujos piroclásticos, que en casos de erupciones anteriores no han llegado a la ciudad, debido a su ubicación en la falda del volcán y la dirección del viento (J.L. Freire, entrevista, 6 de julio 2020)

A largo plazo, el caso del volcán Tungurahua representa el accionar concreto de las instituciones responsables del cuidado y alerta de la población, quienes de diferentes maneras han actuado de acuerdo a sus propios intereses. Pese a ser un ejemplo de gestión inadecuada, con un enorme impacto sobre las poblaciones locales, en particular mujeres rurales y sus modos
2. Al respecto el documentalista Juan Carlos Travieso Fajardo (realizador) y el periodista Martin Oller Alonso (productor) realizaron el documental "Los ojos del volcán" (2020), donde narran la odisea de los pobladores de Baños en el retorno a sus hogares: https:// culturasperiodisticas.com/2020/o1/o7/ corto-documental-ojos-del-volcan/ 
de vida de sustento, y si bien generó reflexiones y cambios importantes en la institucionalidad del Ecuador, estos nunca se enfocaron "genuinamente en la mejora de las vulnerabilidades, incluyendo la mitigación y prevención, a largo plazo" (C. Velasco, presidente de la Asociación de Profesionales en Gestión de Riesgos, 14 de abril 2021, comunicación personal). Y esto se da pese al impacto de El Niño, de 1997/1998, que, debido a sus efectos macroeconómicos, sí provocó cambios institucionales ${ }^{3}$ y una cierta apertura, tanto con los discursos, como con las prácticas internacionales en la gestión de riesgos, según lo confirmado por la exdirectora de la Secretaría Nacional de Gestión de Riesgos, M. P. Cornejo (comunicación personal, 10 de octubre, 2020).

La noche del 16 de abril de 2016, Ecuador sufrió un terremoto de 7,8 MW, dejando 663 fallecidos, 6.247 personas heridas, 28.775 refugiados en albergues establecidos y gestionados por las Fuerzas Armadas (FF.AA.), la mayoría manteniéndose bajo régimen militar durante 12 a 20 meses (provocando en varios casos agresiones sexuales y relaciones clientelares para obtener donaciones y otros favores, véase Waldmüller et al., 2018); además de la destrucción de más de 20.000 viviendas, edificios e infraestructura (vial, telecomunicaciones, redes de electrificación, servicios básicos) y diversas afectaciones en cantones de diez provincias. Se han analizado los acontecimientos en publicaciones anteriores (Waldmueller et al., 2019; Waldmüller et al., 2018; Waldmüller \& Nogales, 2021), en las cuales se realizó un seguimiento etnográfico y documentario en dos provincias (Manabí y Esmeraldas) a lo largo de tres años.

En resumen y para fines argumentativos, es importante destacar en adelante algunas medidas que se tomó durante la presidencia de Rafael Correa con respecto a la gestión de riesgos, que en su totalidad encadenó una serie de indagaciones por parte de la Fiscalía y Contraloría nacional, aún inconclusas, respecto al millonario abuso y corrupción con los fondos públicos (El Universo, 2018), incluyendo la falta de respuestas y finalización de importantes obras durante la "reconstrucción" posdesastre. Como respuesta a esta catástrofe se puso en marcha un amplio proceso biopolítico, logístico, económico y emocional orientado hacia la reconstrucción costeña, bajo el recién formado y llamativo Comité de Reconstrucción, presidida por el entonces Vicepresidente Jorge Glas, quien años más tarde fue sentenciado a 6 años de cárcel por asociación ilícita, en el desorbitado entramado de corrupción conocido como "Caso Odebrecht". La creación del Comité implicó un cambio total en la institucionalidad de la gestión de desastres, quitándole capacidad de decisión a la Secretaría Nacional de Gestión de Riesgos (SNGR), minimizando la necesaria transparencia en el uso de los fondos asignados y el control democrático, y volviendo a la politización opaca de la gestión como en tiempos anteriores.

En rasgos generales se hizo rápidamente visible, entre mayo y julio de 2016 , que este proceso tomó una forma y orientación particular, al contrario de algunas experiencias históricas y estándares internacionales recientes (Pallares, 2016). Adicionalmente, se reinstauró el dominio de los militares con respecto a la respuesta inmediata en el establecimiento de los albergues y el almacenamiento y gestión las donaciones, nacionales e internacionales, destinadas a los damnificados. Similar a la gestión militar del Tungurahua, son abundantes las acusaciones de saqueo de hogares, privación de donaciones alimentarias y creación de redes clientelares (incluyendo favores sexuales) para obtener dichas donaciones (Vega et al., 2019).

De manera general, se lanzó un amplio proceso fiscalizador que, por un lado, buscó restaurar el orden y el control sobre las poblaciones y territorios, aun incluyendo una serie de violaciones de derechos básicos; y por otro lado, en muchos casos se modificó el régimen de tenencia de casas y tierras, obligando a determinadas poblaciones a entregar sus tierras al Estado, a cambio de recibir una pequeña vivienda, de las cuales muchas de ellas nunca tuvieron los respaldos legales de registro de propiedad. Según las palabras de la ex-directora del Ministerio de Desarrollo Urbano y Vivienda (MIDUVI) para la provincia de Esmeraldas (comunicación personal, 3 de abril 2017), responsable para las viviendas, la situación sirvió para "aprovecharse de la reconstrucción para poner orden", pues se iban a modificar los núcleos familiares (extendidos entre las familias afroecuatorianos) bajo un mismo techo.

Es así que, por ejemplo, la isla de Muisne, en la provincia de Esmeraldas, quedó bajo asedio gubernamental y militar durante más de 24 meses, por la decisión del gobierno de desalojar la isla por completo (aproximadamente 9000 habitantes). De manera similar, la isla de Portete fue evacuada en gran parte y, tanto terrenos como agua potable se entregaron a un complejo hotelero perteneciente a una cadena internacional. No es casual, desde una perspectiva decolonial interna,
3. Entre ellos, la participación de Ecuador en el Comité Nacional para e Estudio Regional del Fenómeno de El Niño (ERFEN). 
que en ambos casos la población doblemente afectada es mayoritariamente afrodescendiente (Waldmueller, 2020; Waldmüller et al., 2019).

Siguiendo el ejemplo de las nuevas urbanizaciones y viviendas que el MIDUVI construyó en respuesta a la erupción del Tungurahua, el Estado se benefició con el emplazamiento de urbanizaciones durante el prolongado estado de emergencia dictado para las provincias costeñas. Bajo el régimen de emergencia, las contrataciones públicas pueden ocurrir de forma directa, sin convocatoria oficial respectiva, y sin la debida fiscalización. Es decir, se permitió la canalización de elevados fondos públicos (entre 2016 y 2019 superaron un valor total de 1.5 billones de dólares) hacia sectores afines al gobierno nacional o local de turno, y, en consecuencia, con la capacidad de transformar los paisajes de poder económico y político a largo plazo.

Si bien el MIDUVI construyó miles de viviendas nuevas en respuesta al terremoto, y se siguió, en principio, el ejemplo del Tungurahua, se proyectaron viviendas desde un imaginario sumamente urbano, en estrechas urbanizaciones sin jardines, sin espacio para la agricultura familiar de subsistencia y en total desconocimiento de las particularidades culturales (extensiones familiares, inseguridad vecinal) y climáticas de la Costa. Las casas entregadas, a crédito de 1000 dólares por persona, tienen una extensión de solo 40 metros cuadrados, en general son de baja calidad (por ejemplo, con techos que no protegen durante las temporadas de lluvias), como resultado de ello, a finales del 2019 un sinnúmero de urbanizaciones se encontró casi o completamente abandonadas 0 , irregularmente, en venta.

En el caso de la erupción volcánica del Tunguharua, si bien el Estado se ocupó de la construcción de casas amplias y de mejor estructura (Toro Mayorga, 2020), se organizó la reubicación de cientos de familias a nuevas comunidades recién creadas, muchas de ellas en zonas de riesgo inminente, afectadas por la ocasional caída de cenizas. Según nuestros interlocutores, la ubicación de dichas urbanizaciones no siguió la motivación de proporcionar protección y bienestar para las familias afectadas, sino, igual que en el caso del terremoto, se tomaron acciones para beneficiar, a través de compra-ventas públicas de terrenos con sobreprecio, a ciertas personas o grupos con cercanía a los gobiernos locales, así como aumentar o disminuir el número poblacional en los distritos electorales, o bien para que ciertos gobiernos locales puedan acceder a fondos y obras públicos. Otra lógica precedente es la de cambiar recintos y zonas rurales o urbanos, según intereses políticos, para crear o fusionar las entidades políticas de la administración pública.

Estas lógicas han sido fundamentales en vista de las elecciones presidenciales a inicios de 2017, donde se decidió la (supuesta) continuidad del proyecto "correista" (2007/8-2017) mediante un posible sucesor, al haberse culminado las posibilidades de presentarse como candidato del entonces presidente Rafael Correa. Efectivamente, en las elecciones ganó el candidato oficial, Lenin Moreno, en gran parte gracias a los votos de la provincia de Manabí, siendo la tercera más poblada del país, lo cual se relaciona fuertemente con políticas de rumores y prácticas clientelares para fortalecer el apoyo partidario a cambio de recibir donaciones, viviendas, créditos, etc. Abundan casos documentados de obras prometidas a los que demostraron visiblemente su apoyo al partido, pero nunca entregadas, obras entregadas, pero nunca terminadas; entregas de viviendas y urbanizaciones a gente ajena a la zona y que no se vio afectada (Arévalo Gross, 2018); y sobre todo, el incumplimiento de obras destinadas a cubrir necesidades básicas (agua potable, saneamiento y alcantarillado) cuya falta histórica ha sido clave para la ocurrencia del desastre social después el terremoto (Bravo, 2018; González et al., 2018; Medina, 2017; Ochoa, 2017). Todo esto bajo vigilancia de las FF.AA., y muchas veces a costo de los sectores más vulnerables y pobres (por ejemplo, en las zonas marginales de poblaciones turísticas relevantes), marcados por diferencias étnicas, económicas y de género.

Para mencionar un ejemplo sobresaliente, luego del terremoto se sucedió la regularización y ampliación de concesiones camaroneras a lo largo del perfil costero, muchas veces ilegalmente ubicadas en áreas de protección ambiental, sobre todo de manglares, bajo el afán de canalizar fondos a los "sectores productivos" (El Telégrafo, 2017). Las empresas camaroneras proveen fuente de empleo solo para algunos puestos altamente precarizados y temporales, ocupados - caso de la provincia de Esmeraldas - por afrodescendientes, y ubicados en lugares muchas veces tan alejados y de difícil acceso que los niños no pueden seguir su instrucción escolar. Además, destruyen y contaminan el ambiente y la pesca por haberse expandido sobre las zonas de manglares (C-CONDEM, 2017; Ocampo-Thomason, 2006), un bioma considerado de 
alta importancia en el equilibrio del clima global. A pesar de ello, las exportaciones camaroneras superaron a las de banano entre 2016 y 2017 (Serrano Moscoso, 2018), constituyéndose hoy en día como el segundo sector económico más importante del país. La próxima sección busca contextualizar estos acontecimientos a la luz del cambiante rol de las FF. AA y la institucionalidad pública en el contexto internacional de la GdR.

\section{RASTREO DEL ROL DE LAS FF. AA EN LA GDR}

En vista de lo expuesto anteriormente, es importante reconocer los principales cambios internacionales que se dieron con respecto a la atención sobre los riesgos, y como éstos incidieron en las instituciones públicas, incluyendo a las FF. AA. durante el periodo en cuestión. En la actualidad, las FF. AA. recuperaron, tras los desastres, mucho del poder anterior, cercano al poder ejecutivo de turno.

Entre 1990 y 1999 la Organización de las Naciones Unidas (ONU) declaró la Década Internacional para la Reducción de los Desastres Naturales (DIRDN), que tuvo por objetivo "reducir, por medio de una acción internacional concertada, especialmente en los países en vías de desarrollo, la pérdida de vidas, los daños materiales y trastornos sociales y económicos causados por los desastres naturales" (ONU, 1989, p.15). La perspectiva es afín a un modelo de desarrollo que favorece el nivel multilateral e internacional, puesto que considera que "el impacto de los desastres naturales representa un atraso en el desarrollo y una carga demasiado grande para los países más vulnerables y pobres" (Valdéz, 1997, p. 5). Por ejemplo, por parte de la cooperación internacional, en el año 2007, destacan fondos importantes para el Cantón Penipe, una de las poblaciones más afectadas por el Tungurahua. Destinados al Programa de Reasentamiento, fueron administrados por la fundación ecuatoriana Esquel (Fondos Internacionales para la Atención a Desastres, 2010). De igual manera han sido cruciales los aportes internacionales y bilaterales para atender el terremoto de 2016, sin embargo, fueron canalizados a través de la Secretaría Técnica de Cooperación Internacional, cuyo cuestionado accionar permitió la incidencia del Estado como actor principal en la gestión de riesgos.

A nivel internacional, Naciones Unidas (ONU), a través de la Oficina de las Naciones Unidas para la Reducción del Riesgo de Desastres (UNDRR y el Secretariado de la Estrategia Internacional para la Reducción de Desastre son encargadas a unificar criterios entre países, para ello han organizado una serie de reuniones, en las cuales el Ecuador ha participado y suscrito acuerdos internacionales. Destacan el Acuerdo de Yokohama en 1994, donde se trabajó con prevención y mitigación; el Marco de Acción de Hyogo, realizada en el año 2005, donde principalmente se abordó el aumento de la resiliencia; y finalmente el Marco de Acción Sendai (2015), donde se establece un programa de acción en prevención y mitigación hasta el año 2030. Asimismo, Ecuador, junto con Bolivia, Colombia, Perú y Venezuela, integra el Comité Andino para la Prevención y Atención de Desastres (CAPRADE), desde el año 2002 (Rodriguez y Toulkeridis, 2019).

A la luz de la Constitución de la República del Ecuador y de la Ley Orgánica de la Defensa Nacional, y dada la ausencia de una ley de gestión de riesgos en el caso del Ecuador, así como por los altos niveles de riesgo a los que se encuentra expuesto, la prevención y la gestión de riesgo son aspectos esenciales dentro de la administración gubernamental, resaltando por el rol que todavía desempeñan a las FF. AA.

Uno de los pilares fundamentales en que se sustenta la seguridad y defensa de una nación, lo constituye sin lugar a dudas la capacitación y sensibilización que poseen los integrantes de las FF. AA en la atención a la población y la seguridad ciudadana. Elemento que se refleja en la Carta Magna del Ecuador de 2008, en cuyos artículos 158 y 162, define la misión fundamental de las FF. AA en términos de la defensa de la soberanía y la integridad territorial, y son descriptas como una institución cuyo objetivo es la protección de los derechos, libertades y garantías de los ciudadanos. El artículo 389 de la Constitución establece que el Estado protegerá a las personas, las colectividades y la naturaleza frente a los efectos negativos de los desastres de origen natural 0 antrópico mediante la prevención ante el riesgo, la mitigación de desastres, la recuperación y mejoramiento de las condiciones sociales, económicas y ambientales, con el objeto de minimizar las condiciones de vulnerabilidad. También la Ley de Seguridad Pública y del Estado (2009), en su artículo 11, señala que "la prevención y las medidas para contrarrestar, reducir y mitigar los riesgos de origen natural y antrópico o para reducir la vulnerabilidad, corresponden a las entidades públicas y privadas, nacionales, regionales y locales. La rectoría la ejercerá el Estado a través 
de la Secretaría Nacional de Gestión de Riesgos" (p.6). Efectivamente, resulta fragmentado y disperso el marco normativo-legal relevante para la gestión de riesgos, cuya orientación principal, pese a años de discusiones y demandas por parte de expertos y de la sociedad civil, nunca ha sido plasmada en una concreta ley de gestión de riesgos. Esta omisión negligente permite la constante subordinación a lógicas e intereses divergentes de las poblaciones afectadas y amenazadas. En consecuencia, no es de sorprender que se ha convertido a la SNGR en una nueva entidad de "servicio nacional", de dudosa representación de la institucionalidad nacional, dado que no figura en la Constitución vigente del 2008 y que, a nuestro parecer, expresa la recurrente institucionalización del colonialismo interno mediante la (mala) gestión de los riesgos.

En el caso del volcán Tungurahua, la actuación de las FFAA, además de organizar la evacuación, impidió durante cuatro meses que la gente regrese a sus casas. Esta situación aumento la vulnerabilidad económica de los pobladores de Baños, dado el abandono forzado de hogares y escuelas, comercios y cultivos (luego destruidos por los flujos piroclásticos), sumado a la desidia del gobierno para dar una respuesta adecuada, entendiendo que los pobladores quedaron a la deriva en sus necesidades cotidianas. Sin apoyo económico y sin la suspensión del cobro de deudas, fueron recibidos por familiares 0 amigos en ciudades cercanas, como Ambato, Latacunga y Riobamba. Es de entender que las más de 16000 personas evacuadas sufrieron una doble vulnerabilidad, no solo por no contar con planes de prevención (que en poblaciones rurales debe incluir animales de granja) sino también por encontrarse en situaciones de inseguridad laboral y económica (J.L. Freire, entrevista, 6 de julio 2020). La tensión generada eclosionó el 5 de enero de 2000 , momento en que los habitantes se enfrentaron a la fuerza militar y, finalmente, retoman su ciudad. Esto sucedió a pocos días del congelamiento de fondos y ahorros privados de los ecuatorianos, y la forzada conversión del sucre nacional al dólar estadounidense con todo el desastroso impacto socioeconómico para las poblaciones. En palabras del exalcalde de Baños, (J.L. Freire, entrevista, 6 de julio 2020), "...había una convulsión terrible, la gente decía que el tema Baños era una cortina para que la gente no hable del tema económico, de lo que estaba pasando".

\section{CONCLUSIONES}

Este artículo tuvo por objetivo poner en contexto económico y político más amplio los casos de la erupción del Tungurahua (1999-2006) y del terremoto en el litoral ecuatoriano (2016) desde una mirada decolonial interna, con el fin de vislumbrar la trayectoria que tomaron las instituciones involucradas, la producción de conocimiento "oficial" y, sobre todo, el rol de las FF.AA. desde los años 90 con respecto a la GdR. El argumento principal que sostiene este análisis es que, si bien hubo ciertos cambios institucionales y avances importantes en Ecuador y la región, todavía prevalece un sesgo modernista en la atención de los riesgos y desastres, con un marcado acento técnico-científico, que deja de lado el aspecto sociocultural o psicológico que afecta a las poblaciones más vulnerables, y por lo tanto es instrumentalizador, prolongando el colonialismo interno, en clave étnica y de género, en el sentido de subordinar el bienestar de poblaciones, y territorios a los intereses políticos o económicos de turno.

Históricamente, el Estado ecuatoriano poscolonial ha desempañado en un rol paternalista, cuyas Fuerzas Armadas, además de representar el uso legal de la fuerza legítima al estilo weberiano, también ejercen el uso legal del orden, muchas veces con una mirada alejada de los conocimientos tradicionales de las comunidades, imponiendo sistemas de acción jerárquica que representan al Estado moderno. En este sentido, la percepción de las necesidades de la población vulnerable ante eventos naturales y antrópicos, entendiendo la urgente reducción de la exposición al riesgo, representa frecuentemente una mirada clasista, racista y profundamente desigual, propia del Estado Nación poscolonial moderno.

En los casos estudiados, esta mirada se profundizó con el contexto de la crisis económica y política, que evidencia la falta de la voluntad política de, por un lado, manejar recursos de forma transparente, y por otro, de atender a las vulnerabilidades interseccionales con enfoque en los derechos humanos, de manera participativa y por lo general, valorando los conocimientos y perspectivas locales (Lavell, 2006; Lavell \& Franco, 1996). La histórica correlación entre grandes eventos naturales y crisis políticas llevó a la reflexión sobre la utilización política de estos eventos para desviar la atención de los problemas económicos, sin perder de cuenta que la complejidad de la gestión de riesgos tiene relación con la construcción histórica basada en principios esencialmente jerárquicos y científicos, que se han centrado en la respuesta técnica inmediata al desastre y 
no han permitido la generación de procesos de prevención sociocultural, dada que la gestión de riesgos ha sido considerada un tema de segundo orden de importancia, en un país atravesado por crisis políticas constantes.

En este sentido, frente a los estallidos del volcán Tungurahua en 1999 y del terremoto costeño de 2016, constatamos que la intervención militar no se realizó primariamente para proteger a la población, sino para evitar actos de insurrección pública frente a la crisis política y económica del momento, así como para cubrir la ineficaz forma de ejecutar la ayuda destinada a la mitigación de los daños. En definitiva, pese a confirmar al ejército como garante de la estabilidad nacional, en ambos casos, la gestión de riesgos acarrea violaciones de derechos humanos, contradicción que es afín al colonialismo interno, dado que parte de las comunidades afectadas pertenecen a etnias históricamente relegadas, tanto los indígenas campesinos, como los afrodescendientes costeños, en un contexto donde la gestión territorial es un elemento fundamental en el ordenamiento territorial del Estado. Asimismo, la institucionalidad militar es, igual al Estado poscolonial, intrínsecamente patriarcal (Colectivo Miradas Críticas del Territorio desde el Feminismo, 2017) y tiende a promover conductas violentas que agregan un factor de vulnerabilidad en clave de género y edad.

Concluimos reiterando que el paso hacia la salida de la continuada autocolonización requiere para la gestión de riesgos un marco normativo-legal e institucional profundamente democrático, ello implica que la población sea actor empoderado y participante de planes de educación, prevención y mitigación que les permita tener autonomía de decisión y capacidad financiera para enfrentarse a eventos naturales o antrópicos.

\section{REFERENCIAS}

Amin, S. (1975). El desarrollo desigual, ensayo sobre las formaciones sociales del capitalismo periférico. Fontanella.

Andolina, R., Laurie, N., \& Radcliffe, S. A. (2009). Indigenous Development in the Andes: Culture, Power, and Transnationalism. Duke University Press.

Andrade-Vásquez, M. (2021). Doctrina y estructura militar ecuatoriana: De cara o de espaldas a las amenazas del siglo XXI. URVIO. Revista Latinoamericana de Estudios de Seguridad, 29, 109-121. https://doi.org/10.17141/urvio.29.2021.4322

Ardaya Salina, G. (2019). Corrupción e impunidad en el Ecuador de la década perdida. Plataforma por la defensa de la Democracia y los Derechos Humanos en el Ecuador.

Arévalo Gross, L. (2018). Promesas rotas. GKill. https://gk.city/2018/04/19/ fondos-reconstruccion-terremoto-perdernales-gobierno/

Atallah, D. G. (2016). Toward a decolonial turn in resilience thinking in disasters: Example of the Mapuche from southern Chile on the frontlines and faultlines. International Journal of Disaster Risk Reduction, 19, 92-100. https://doi.org/10.1016/j.ijdrr.2016.08.027

Atallah, D. G., Bacigalupe, G., \& Repetto, P. (2019). Centering at the Margins: Critical Community Resilience Praxis. Journal of Humanistic Psychology, o02216781882530. https://doi. org/10.1177/0022167818825305

Bravo, E. (Ed.). (2018). Ecología política de los desastres. El terremoto del 2016 en Ecuador. Universidad Politécnica Salesiana \& Abya Yala.

Büscher, B., Feola, G., Fischer, A., Fletcher, R., Gerber, J.-F., Harcourt, W., Koster, M., Schneider, M., Scholtens, J., Spierenburg, M., Walstra, V., \& Wiskerke, H. (2021). Planning for a world beyond COVID-19: Five pillars for post-neoliberal development. World Development, 140, 105357. https://doi. org/10.1016/j.worlddev.2020.105357

Cardona, O. D. (2008). The Need for Rethinking the Concepts of Vulnerability and Risk from a Holistic Perspective: A Necessary Review and Criticism for Effective Risk Management. En G. Bankoff, G. Frerks, \& D. Hilhorst (Eds.), Mapping Vulnerability (pp. 37-52). Earthscan.

Carrigan, A. (2015). Towards a Postcolonial Disaster Studies. En E. DeLoughrey, J. Didur, \& A. Carrigan (Eds.), Global Ecologies and the Environmental Humanities (pp. 117-140). Routledge, Taylor \& Francis Group.

C-CONDEM. (2017). Certificando la destrucción. La industria camaronera en el Ecuador. En E. Bravo, M. Moreano, \& I. Yánez (Eds.), Ecología política en la mitad del mundo. Luchas ecologistas y reflexiones sobre la naturaleza en el Ecuador (pp. 235-251). Abya-Yala. 
Cielo, C., \& Vega, C. (2015). Reproducción, mujeres y comunes Leer a Silvia Federici desde el Ecuador actual. Nueva Sociedad, 256 (marzo-abril).

Colectivo Miradas Críticas del Territorio desde el Feminismo. (2017). (Re)patriarcalización de los territorios. La lucha de las mujeres y los megaproyectos extractivos. Ecología Política, 54, 65-69.

Collins, P. H., \& Bilge, S. (2016). Intersectionality. Polity Press.

Crenshaw, K. (1989). Demarginalizing the Intersection of Race and Sex: A Black Feminist Critique of Antidiscrimination Doctrine, Feminist Theory and Antiracist Politics. The University of Chicago Legal Forum, 140, 139-167.

El Telégrafo. (2017, marzo 16). 200 camaroneras en Muisne operan bajo concesiones gubernamentales renovables. El Telégrafo, en línea. https://www.eltelegrafo.com.ec/noticias/ economia/8/20o-camaroneras-en-muisne-operan-bajo-concesiones-gubernamentales-renovables

El Universo. (2018, septiembre 1). Dudas por uso de fondos del terremoto para otras cuentas. El Universo, 4 .

Escobar, A. (2002). "Worlds and Knowledges Otherwise”: The Latin American modernity/coloniality Research Program. http://www.unc.edu/ aescobar/text/eng/Worlds_and_Knowledges_Otherwise. doc

Fanon, F. (1966). The Wretched of the Earth. Grove Press.

Fanon, F. (1967). Black Skin White Masks. Grove Press.

Federici, S. (2014). Revolución en punto cero. Trabajo doméstico, reproducción y luchas feministas. Traficantes de Sueños.

Fernández, A.G., Waldmüller, J., \& Vega, C. (2020). Comunidad, vulnerabilidad y reproducción en condiciones de desastre. Abordajes desde América Latina y el Caribe. Presentación del dossier. Íconos - Revista de Ciencias Sociales, 66, 7-29. https://doi.org/10.17141/iconos.66.2020.4156

GAD Baños de Agua Santa. (2014). Plan de Desarrollo y Modelo de Gestión 2014-2019. GAD Baños de Santa Agua.

García Osorio, N. (2013). La crisis financiera del Ecuador, 1998-20oo. Economía y Negocios, 4(1), 5. https://doi.org/10.29019/eyn.v4i1.16o

González, P., Pacheco, M., \& Bonilla, M. (2018, mayo 10). Esmeraldas tiene poco acceso al agua y al alcantarillado. El Comercio, en línea.

González Casanova, P.G. (1965). Internal colonialism and national development. Studies in Comparative International Development, 1(4), 27-37.

Hannigan, J. A. (2012). Disasters without borders: The international politics of natural disasters. Polity Press.

Kofman, E. (2016). Repensar los cuidados a la luz de la reproducción social: Una propuesta para vincular los circuitos migratorios. Investigaciones Feministas, 7(1), 35-56.

Konyndyk, J., \& Aly, H. (2021, enero 11). Decolonizing Aid: Rethinking Humanitarianism Episode 7. https://www.cgdev.org/blog/decolonizing-aid-rethinking-humanitarianism-episode-7

Lavell, A. (2006). Apuntes para una reflexión institucional en países de la Subregión Andina sobre el enfoque de la Gestión del Riesgo. CAF-PREDECAN.

Lavell, A., \& Franco, E. (Eds.). (1996). Estado, sociedad y gestión de los desastres en América Latina. En busqueda del paradigma perdido. FLACSO/La RED/ITDG.

Lebret, L.J. (1967). Dynamique concrète du développement. Éditions Ourières.

Lyotard, F. (1979). La condition postmoderne: Rapport sur le savoir (The Postmodern Condition: A Report on Knowledge) (G. Bennington \& B. Massumi, Trads.). Minnesota University Press.

Marchezini, V. (2015). The Biopolitics of Disaster: Power, Discourses, and Practices. Human Organization, 74(4), 362-371. https://doi.org/10.17730/0018-7259-74.4.362

Medina, A. (2017, junio 6). Contraloría determinó que fondo asignado a damnificados del terremoto se usó en otros rubros. El Comercio, en línea. http://www.elcomercio.com/actualidad/contraloriafondo-damnificados-terremoto-ministeriodelinterior.html

Moraña, M., Dussel, E., \& Jáuregui, C. A. (2008). Coloniality at Large: Latin America and the Decolonial Debate. Duke University Press. 
Morozov, E. (2013). To Save Everything, Click Here. Technology, Solutionism and the Urge to Fix Problems That Don't Exist. Allen Lane.

Müller, M.-M. (2020). Enter 9/11: Latin America and the Global War on Terror. Journal of Latin American Studies, 1-29. https://doi.org/10.1017/Soo22216X20000565

Ocampo-Thomason, P. (2006). Mangroves, People and Cockles: Impacts of the Shrimp- Farming Industry on Mangrove Communities in Esmeraldas Province, Ecuador. En C. T. Hoanh, T. P. Tuong, J. W. Gowing, \& B. Hardy (Eds.), Environment and Livelihoods in Tropical Coastal Zones: Managing Agriculture-Fishery-Aquaculture Conflicts (pp. 140-153). CAB International.

Ochoa, D. (2017). Visión antropológica del desastre: El caso de Canoa un año despues el terremoto. En A. Carrión, I. Giunta, A. Mancero, \& G. Jiménez (Eds.), Posterrmoto, gestión de riesgos y cooperación internacional: Ecuador (pp. 211-231). IAEN.

Pallares, M. (2016, abril 30). El Comité de Reconstrucción... es el mismo gobierno. Cuatro Pelagatos. http://4pelagatos.com/2016/o4/30/el-comite-de-reconstruccion-es-el-mismo-gobierno/

Rebotier, J. (2016). El riesgo y su gestión en Ecuador: Una mirada de geografía social y política (Primera edición). Centro de Publicaciones, Pontificia Universidad Católica del Ecuador.

Serrano Moscoso, S. (2018, enero 13). El producto estrella de 2017. Tierra y Mar. Un espacio para el productor (El Telégrafo), 3.

Timmermann, C. (2020). Epistemic Ignorance, Poverty and the COVID-19 Pandemic. Asian Bioethics Review. https://doi.org/10.1007/s41649-020-00140-4

Toro Mayorga, L.I. (2020). Tras las cenizas y el sismo: Vivienda en la Sierra rural de Ecuador y Perú. FLACSO Ecuador.

Vargas, J. (2009). La Plurinacionalidad: Un paradigma de transformación social. En A. Acosta \& E. Martínez (Eds.), Plurinacionalidad. Democracia en la diversidad (pp. 99-107). Abya Yala.

Vega, C., Paredes, M., \& Almeida, A. N. (2019). Desigualdades y crisis reproductiva tras el terremoto en la costa ecuatoriana. Estrategias familiares ante el modelo de desarrollo y trabajo extractivo. Revista de Antropología Iberoamericana, 14(2), 323-350. https://doi.org/10.11156/aibr.140208

Waldmueller, J. M. (2020). (In)Visibilization through Decolonial Delinking? Disrupting the Permanently Neglected Disaster at the Border of Colombia and Ecuador. Disaster Prevention and Management, 29(6), 929-942. https://doi.org/10.1108/DPM-01-2020-0002

Waldmueller, J. M., Nogales, N., \& Cobey, R. J. (2019). Assessment of local adaptive capacities in the context of local politics after the 2016 Ecuadorian earthquake. International Journal of Disaster Risk Reduction, 35, 101062. https://doi.org/10.1016/j.ijdrr.2019.01.001

Waldmüller, J. M., Jamali, H., \& Nogales, N. (2019). Operationalizing Sustainable Development Goals in Vulnerable Coastal Areas of Ecuador and Pakistan: Marginalizing Human Development? Journal of Human Development and Capabilities, 20(4), 468-485. https://doi.org/10.108o/19452829.2019.16668 10

Waldmüller, J. M., \& Nogales, N. (2021 en prensa). La noche que tembló Ecuador. Una mirada a la reconstrucción y recuperación: Camino a la prevención y resiliencia. Abya-Yala.

Waldmüller, J. M., Nogales, N., Cobey, R., \& Urigüen, M. C. (2018). Monitoreo de derechos humanos y gestión de riesgo desde un enfoque del desarrollo humano: Visibilizar e invisibilizar actores, derechos y capacidades. FORO, 30(II cuatrimestre), 5-30.

Walsh, C. (Ed.). (2004). Pensamiento crítico y matriz (de)colonial. Reflexiones latinoamericanas. Abya Yala.

Walsh, C. (2009). Interculturalidad, estado, sociedad. Luchas (de)coloniales de nuestra época. Abya Yala.

Watson, S. D. (2019). International Order and the Politics of Disaster. Routledge. https://doi. org/10.4324/9780429259272

Williamson, J. (1993). Development and the "Washington Consensus". World Development, 21, 1239-1336.

Yépez, V., Toledo, J., \& Toulkeridis, T. (2020). The Armed Forces as an Immediate Response State Institution and Its Participation as an Articulator in the Risk Management in Ecuador. En Á. Rocha, M. Paredes-Calderón, \& T. Guarda (Eds.), Developments and Advances in Defense and Security (Vol. 181, pp. 545-554). Springer Singapore. https://doi.org/10.1007/978-981-15-4875-8_47 